\title{
End-to-end QoS Signaling Protocol and its Performance Analysis for LTE Networks
}

\author{
Géza Horváth and Péter Fazekas, Member, IEEE
}

\begin{abstract}
In order to effectively deliver traffic from different applications, providing end-to-end Quality of Service (QoS) is critical in Long Term Evolution (LTE) networks. Mobility requires special handling of QoS enforcement rules and methods. The LTE QoS Signaling (LQSIG) protocol presented in this paper will allow ensuring resource reservation before using a data path. Operation of the proposed protocol in different mobility scenarios is also explained. The key features of the protocol include LTE QoS model mapping to QSPEC (QoS Specification) objects used in reservation and interworking with mobility protocols in the LTE protocol stack, especially with Radio Resource Control (RRC). The basics of an analytical model are proposed in order to determine the blocking probability at the bottlenecks of LTE network. A performance analysis of LQSIG describes its properties compared to legacy LTE networks.
\end{abstract}

Keywords-LTE, End-to-end QoS, Resource Reservation.

\section{INTRODUCTION}

A LL the LTE network elements are handling traffic and delivering services in a heterogeneous environment. Even the network itself can be heterogeneous: vendors may implement different network components with different technologies included or build up various protocol stacks. An LTE network is characterized by the separated control plane $(\mathrm{CP})$ and user plane (UP) and support of all IP-based services. A legacy underlying network-layer can be used to have circuit switched voice service fallback facility. As shown in Fig. 1, three different network domains can be distinguished in the Evolved Packet System (EPS): an access network with radio interfaces (eUTRAN), a backhaul network to aggregate traffic and core network (CN) for mobility management and UP traffic processing. User equipments (UEs) and evolved NodeBs (eNodeBs) are operating in eUTRAN. A Mobility Management Entity (MME) is responsible for signaling only, located in $\mathrm{CN}$,

Paper received April 2, 2014; revised July 11, 2014; accepted July 16, 2014. Date of publication July 31, 2014. The associate editor coordinating the review of this manuscript and approving it for publication was Prof. Grozdan Petrović.

This paper is a revised and expanded version of the paper presented at the 21th Telecommunications Forum TELFOR 2013.

Géza Horváth is with the Department of Networked Systems and Services, Budapest University of Technology and Economics, Magyar tudósokkörútja 2, 1117, Budapest, Hungary (phone: +36703105569 , email: gezah@hit.bme.hu).

Péter Fazekasis with the Department of Networked Systems and Services, Budapest University of Technology and Economics, Magyar tudósokkörútja 2, 1117, Budapest, Hungary (e-mail fazekasp@hit.bme.hu). while a Serving Gateway (SGW) is taking care of UP traffic switching. A Packet Data Network Gateway (PDN$\mathrm{GW})$ is the edge router towards external packet data networks. A Mobile Backhaul Network (MBH) gives the transmission between the core network and eNodeBs [3].

The variety of services provided across LTE networks has different QoS requirements. It would not be economical to solve such performance problems by overprovisioning network capacity. It has a high business impact if an operator can ensure QoS on a LTE network. Effectively using the finite network resources can cut the cost of every bit of transferred data or second of calls. As the popularity of LTE smart terminals is rising continuously and service providers are porting their applications from former legacy platforms like GSM/UMTS to the new one, LTE will highlight the need for end-to-end QoS requirements to serve various service demands [1], [2].

Numerous QoS signaling protocols have been proposed to solve mobility issues. However, most of them were built upon the Resource Reservation Protocol (RSVP), which was designed for fixed networks, and therefore has some drawbacks in mobile environments. Moreover, most of them turned out to be complex and inefficient in terms of bandwidth utilization. There were some proposals also for adapting signaling protocols to MobileIP (both v4 and v6) but these kinds of solutions do not fit LTE well [5], [11].

The remaining part of this paper is organized as follows. Section II describes the working method of the proposed QoS signaling protocol (called LQSIG).Section III describes the basics of performance evaluation for the proposed method. Section IV concludes the article.

\section{RESOURCE RESERVATION IN LTE WITH LQSIG}

The Next Step in Signaling (NSIS), designed by IETF, is a standardized signaling protocol suite with QoS signaling as a primary use case. The framework consists of two layers. The lower layer, NSIS Transport Layer Protocol (NTLP), is responsible for providing a general transport service for the signaling messages. The General Internet Signaling Transport (GIST) protocol is used in this layer. The signaling messages are originated from the upper layer, NSIS Signaling Layer Protocol (NSLP). As the protocol suite was originally designed to be generic, there is a specified NSLP for ensuring QoS, called QoS NSLP. One of the urgent needs of NSIS is to provide mobility support. In mobile environments frequent handover events may result in degradation of QoS performance [4], [7]. 


\section{A. Resource reservation during the attach procedure}

The LTE QoS Signaling Protocol (LQSIG) proposes immediate interaction with the LTE CP protocol stack. To support this, common interfaces should be provided. On S1, S5 and X2 interfaces it can be matched easily to IP network layer with the help of GIST signaling transport. The challenging part is to adapt LQSIG to RRC on radio interface. LQSIG proposes a hybrid method for interworking with RRC between UE and eNodeB. The protocols behavior is presented through the most significant use cases of LTE, the bearer establishment and handover procedure on X2 interface.

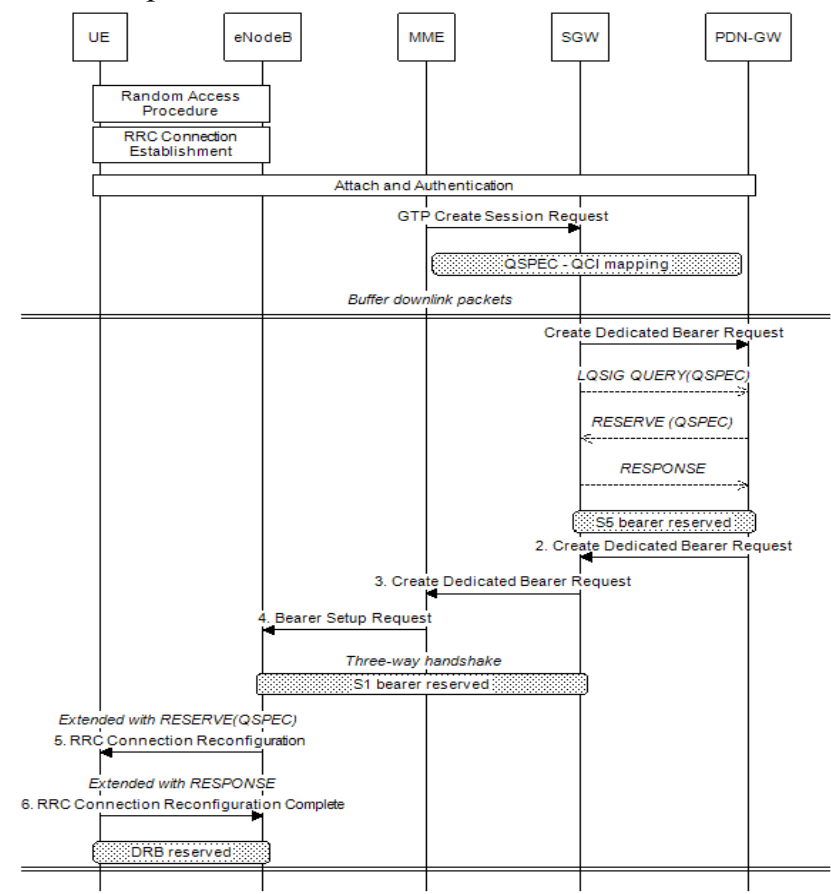

Fig. 1. Bearer establishment with LQSIG.

A bearer establishment can be triggered in several cases, such as initial attach to the network, UE request, networkside request. From resource reservation point of view, all these are the same procedure, so keeping simplicity we only concentrate on the attach procedure. GTP Create Session Request from MME to SGW is triggered by the Update Location Request Answer received by the MME, right after the Random Access Procedure and RRC Connection Establishment phase. At this point, SGW knows about the QoS parameters (ARP, QCI) of the new bearer since these are embedded into the GTP message received from MME.

As S5 bearer is the first part of the EPS bearer to be established, SGW has to initiate a resource reservation on the S5 interface. LTE does not have a solution to complete such reservation, but LQSIG based on QoS-NSLP is able to do so. Our concept for the reservation is based on the three parts of an EPS bearer (DRB (Data Radio Bearer), S1 and S5 bearers) and not making a reservation for the whole path in one phase. As IP is the network layer protocol on S1 and S5 interfaces CP layer, it is easy to use an NSIS GIST-based signaling transport protocol for LQSIG messages [8], [10].

QoS-NSLP defines an RSVP-like RESERVERESPONSE request-acknowledgement pair. RESERVE is the only message that manipulates QoS-NSLP reservation state. RESERVE always contains information about the previous QoS-NSLP message. It can be an explicit confirmation for RESERVE, response to a QUERY or an error code. With a QUERY the initiator can request information about the path without making any reservation. To be accurate, the handshake has to start with a QUERY initiated by the SGW, as it does not have any information about the available resources on the PDN-GW side. With such a three-way handshake between SGW and PDN-GW resources are reserved for the S5 bearer, attach continues with a Create Bearer Request sent from SGW to PDN-GW. S1 bearer resource reservation is very similar to S5.

Resource reservation for the DRB requires the most extensive efforts since IP is not used on $\mathrm{CP}$ of the radio interface. We have only RRC to operate with, therefore a clear interface has to be defined between RRC and GIST protocol layer. A hybrid solution is the extended ASN.1 definition of RRC Connection Reconfiguration and Connection Reconfiguration Complete messages. Both have the possibility to provide this extra content. The extension field allows addition of new fields to the structure without modification of the ASN.1 definition. A non-critical extension may be ignored if it is not recognized, but must be processed if it is recognized. This property gives the opportunity for terminals without LQSIG-capability to operate according to legacy LTE QoS model.

\section{B. QSPEC object mapping to LTE QoS classes}

Every QUERY and RESERVE message of the LQSIG contains the description of the actual QoS model being used. Importing from QoS-NSLP, a so called QSPEC object is sent within the messages. It describes the current resources that are required and depends on the QoS model being used. To be able to transfer LTE QoS model parameters and reserve resources on the path based on QCI values, we define a mapping between QSPEC object and LTE QoS classes as follows.

QSPEC objects have several parameters to describe different QoS models. TMOD-1 is an obligatory parameter, consists of the rate (r), bucket size (b), peak rate $(\mathrm{p})$, minimum policed unit $(\mathrm{m})$ and maximum packet size (MPS) fields. A QSPEC object can have several optional parameters as well, including path latency, path jitter, path packer error and loss rate, preemption and defending priority. Bandwidth was eliminated recently as it can be derived from TMOD-1 field values.

One of the key questions to be answered is how to define the mapping between QSPEC object fields and LTE QoS model. Table 1 presents our proposal for this mapping between QCI classes and QSPEC object identifiers. Four main parameters are assigned to each QCI value: resource type (GBR or non-GBR), priority, packet

delay budget and packet loss rate (PLR). To provide guarantees for bitrates, the operator has to allocate bandwidth on a specific data path. For bandwidth definition in a QSPEC object there is a recommended setting $r=p$ to the peak rate, $b$ and $m$ to some large value. 
TABLE 1: LTE QCI TO LQSIG QSPEC MAPPING

\begin{tabular}{|c|c|c|c|c|c|c|c|c|c|}
\hline \multirow[b]{2}{*}{ Example Service } & \multicolumn{5}{|c|}{ LTE QoS Classes } & \multicolumn{4}{|c|}{ LQSIG QSPEC Object } \\
\hline & 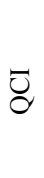 & 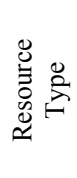 & : & 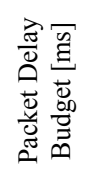 & 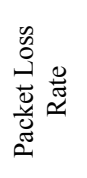 & $\sum_{i}^{\bar{O}}$ & 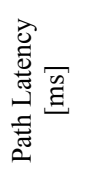 & 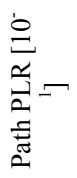 & 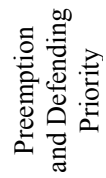 \\
\hline Conversational voice & 1 & \multirow{4}{*}{ 苗 } & 2 & 100 & $10^{-2}$ & \multirow{9}{*}{ 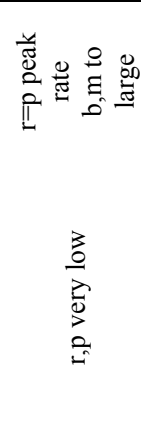 } & 100 & $10^{9}$ & 2 \\
\hline Conversational video (live streaming) & 2 & & 4 & 150 & $10^{-3}$ & & 150 & $10^{8}$ & 4 \\
\hline Real time gaming & 3 & & 3 & 50 & $10^{-3}$ & & 50 & $10^{8}$ & 3 \\
\hline $\begin{array}{l}\text { Non-conversational video (buffered } \\
\text { streaming) }\end{array}$ & 4 & & 5 & 300 & $10^{-6}$ & & 300 & $10^{5}$ & 5 \\
\hline IMS signaling & 5 & \multirow{5}{*}{$\begin{array}{l}\frac{a}{0} \\
0 \\
1 \\
0 \\
z\end{array}$} & 1 & 100 & $10^{-3}$ & & 100 & $10^{8}$ & 1 \\
\hline $\begin{array}{l}\text { Video (buffered streaming) TCP-based } \\
\text { (e.g. www, e-mail, chat, ftp, p2p, file sharing, } \\
\text { progressive video, etc.) }\end{array}$ & 6 & & 6 & 300 & $10^{-6}$ & & 300 & $10^{5}$ & 6 \\
\hline $\begin{array}{l}\text { Voice, video (live streaming), Interactive } \\
\text { gaming }\end{array}$ & 7 & & 7 & 100 & $10^{-6}$ & & 100 & $10^{5}$ & 7 \\
\hline Video (buffered streaming) TCP-based & 8 & & 8 & \multirow[b]{2}{*}{300} & $10^{-3}$ & & \multirow[b]{2}{*}{300} & $10^{8}$ & 8 \\
\hline $\begin{array}{l}\text { (e.g. www, e-mail, chat, ftp, p2p, file sharing, } \\
\text { progressive video, etc.) }\end{array}$ & 9 & & 9 & & $10^{-6}$ & & & $10^{5}$ & 9 \\
\hline
\end{tabular}

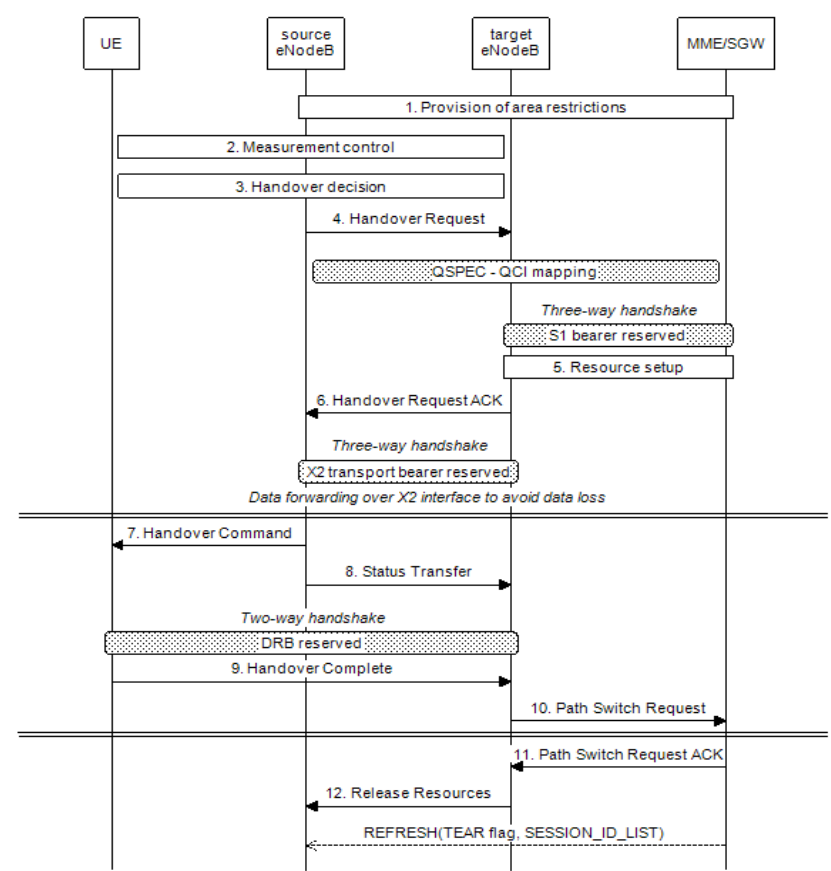

Fig. 2.X2-based handover procedure with LQSIG.

As $\mathrm{r}$ and $\mathrm{p}$ are measured in bytes/sec (including IP header) and the maximum value is 40 terabytes, this method fits any LTE network. Packet delay budget can be converted easily to path latency. It is a 32 bit unsigned integer, measured in msec. This parameter gives the application knowledge on both the minimum and maximum packet delivery delay. Path packet loss rate is also simple to use as PLR is assigned to some QCI value. It is a 32 bit unsigned integer, measured in 10-11 units. It fits properly for the values between 10-2 and 10-6 used in LTE QoS model. Priority assigned to QCI values is considered as a scheduling weight factor for admitted flows. Among QSPEC parameters preemption and defending priority is a good candidate to deal with scheduling. Once a flow is admitted, the preemption priority becomes irrelevant. Instead, its defending priority is used to compare with the preemption priority of the flows. With the help of such mapping, operators can define several QoS classes in addition to standardized ones. If the standard QCI values are not applied, Y.1541 QoS Class parameter could be considered as well [9].

\section{Resource reservation during X2 handover procedure}

In addition to initial bearer establishment mobility, events also have to be considered to realize the properties of LQSIG. An X2 handover is a challenging procedure since one part of the EPS bearer must be kept (S5 bearer), other parts need to be released and rebuilt on a new location (DRB and S1 bearer). As an intermediate step, during this kind of handover we also have X2 transport bearer; resource reservation is also necessary on this section to keep end-to-end QoS requirements.

With Modify Bearer Request DL S1 bearer establishment is triggered, resources must be reserved with three-way handshake initiated from the other side, SGW. Now that all the bearers of the new path are established, traffic has to be terminated on the old one. SGW sends an end marker to source and target eNodeBs to switch the path to the newly established one. Following this a REFRESH message is sent by LQSIG, where TEAR flag is set. All the reserved resources are combined within a SESSION_ID_LIST, included also into the sent REFRESH message. After this step both UL and DL traffic uses the rerouted EPS bearer.

QoS is a critical issue in the operation of today's telecom networks. In this paper we identified the need for providing end-to-end QoS in LTE networks. Based on the findings, we proposed a LTE mobility-aware QoS signaling protocol (LQSIG) with key features such as QSPEC to LTE QoS class mapping, interworking with LTE CP protocol stack, emphasizing the hybrid solution with RRC extension. The proposed analytical model is used to determine the end-to-end blocking probability of LTE networks with LQSIG in use. The performance evaluation - presented in the last section - highlighted that the usage of resource reservation has an advantage compared to standard LTE in terms of blocking probability. Our research plans will include the introduction of more traffic classes into our analytical model. 


\section{Performance EVAluation}

\section{A. Analytical model for $L Q S I G$}

In terms of end-to-end QoS provided via resource reservation, one of the practical measures is to calculate blocking probability. This means the probability that a new request for a bearer cannot be served. We focus our study on S5 interface (between SGW and PDN-GW) since we believe it is a good candidate to be the bottleneck in LTE networks and its optimum utilization is highly economical. Blocking probability on S5 interface is a characteristic property of end-to-end QoS in a LTEenvironment.

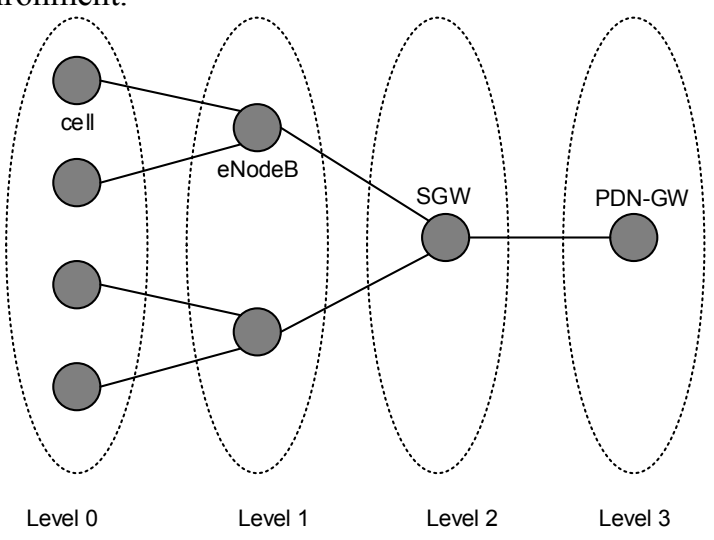

Fig. 3. Analytical model

Our analytical model is a cluster based hierarchical approach, as shown in Fig. 5. Several bearer requests are coming into a cell, forming level 0 clusters. Level 1 is the geographical area of an eNodeB, consists of several level 0 clusters. All clusters on the same level are considered statistically identical because of analytical reasons. A hierarchical model can be continued to SGW and PDNGW level in order to analyze any bottleneck point of LTE networks [6], [12].

Based on the Erlang-B formula, the blocking probability at level $i=0$ is given as

$$
P_{b, 0}=\frac{A_{0}^{c_{0}} / c_{0} !}{\sum_{i=0}^{c_{0}} A_{0}^{i} / i !}
$$

where the offered traffic $A_{0}$ consists of the traffic due to new arrivals, the traffic due to handoffs and the traffic that create stale connection.

$$
A_{0}=\frac{\lambda_{0}+\lambda_{h, 0}}{\mu+n_{0}}+\lambda_{h, 0} d
$$

TABLE 2: PARAMETERS OF THE EQUATIONS

\begin{tabular}{l|c}
\hline Aggregate traffic at level $i$ & $A_{i}$ \\
Number of shared channels at each & $c i$ \\
node of level $i$ & \\
Number of clusters at level $i$ & $N_{i}$ \\
Call duration & $\mu$ \\
Arrival rate at level $i$ & $\lambda_{i}$ \\
Generation rate of handoffs at level $i$ & $\lambda_{h, i}$ \\
Blocking probability at the nodes of & $P_{b, i}$ \\
level $i$ & \\
Flow reservation maintenance time & $d$ \\
without refreshes & \\
\hline
\end{tabular}

The call durations are modeled as independent random variables, following the exponential distribution with parameter $\mu$.Mobile stations' cluster dwell times are modelled as exponentially distributed random variables with parameter $n_{i}$, where $i$ is the cluster level. [13]

At the next level, new traffic is arriving to the eNodeB with $\lambda_{1}=N_{0} \lambda_{0}\left(1-P_{b, 0}\right)$ and the handoff rate is $\lambda_{h, 1}=$ $P_{h, 1}\left(\lambda_{1}+\lambda_{h, 1}\right)\left(1-P_{b, 1}\right)$.

Based on [11], blocking probability on level $i=1$ is given as

where

$$
P_{b, 1}=\frac{A_{1}^{c_{1}} / c_{1} !}{\sum_{i=0}^{c_{1}} A_{1}^{i} / i !}
$$

$$
A_{1}=\frac{N_{0} \lambda_{0}\left(1-P_{b, 0}\right)}{\mu+n_{1}}+N_{0} \lambda_{h, 0} d
$$

On level $\mathrm{i}=2$ we can describe blocking probability with

where the arrival rate

$$
P_{b, 2}=\frac{A_{2}^{c_{2}} / c_{2} !}{\sum_{i=0}^{c_{2}} A_{2}^{i} / i !}
$$

$$
\lambda_{2}=N_{1} \lambda_{1}\left(1-P_{b, 1}\right)=N_{0} N_{1} \lambda_{0}\left(1-P_{b, 0}\right)\left(1-P_{b, 1}\right)
$$

and the offered traffic

$$
A_{2}=\frac{N_{0} N_{1} \lambda_{0}\left(1-P_{b, 0}\right)\left(1-P_{b, 1}\right)}{\mu+n_{2}}+N_{0} N_{1} \lambda_{h, 0} d
$$

Using LQSIG, the aggregate traffic on the S1 interface towards SGW can be described with formula (6). Restricting the number of SGWs to one, $P_{h, 1}=0$, and therefore $\lambda_{h, l}=0$ and $n_{l}=0$. Therefore equation (6) can be simplified as follows.

$$
A_{L Q S I G}=\frac{N_{0} N_{1} \lambda_{0}\left(1-P_{b, 0}\right)\left(1-P_{b, 1}\right)}{\mu}+N_{0} N_{1} \lambda_{h, 0} d
$$

The blocking probability on S5 interface is

$$
P_{b, L Q S I G}=\frac{A_{L Q S I G}^{c_{3}} / c_{3} !}{\sum_{i=0}^{C_{3}} A_{L Q S I G}^{i} / i !}
$$

When using LQSIG resource reservation protocol, we assume that the network is able to serve $C$ parallel calls. We dedicate $S$ out of $C$ to be used for signaling only. It means, that user traffic can use $C-S$ of the total network capacity, $C$; signaling can use the dedicated $\mathrm{S}$ and all the other unused network capacity.

It can be proved easily, that the blocking probability of signaling is lower than the blocking probability of user plane traffic. Our purpose is to maximize the total number of parallel calls. Taking into consideration that blocking of signaling messages means blocking of user traffic as well, we have to concentrate on the blocking probability of the user plane traffic.

A general blocking probability formula on a network using the above described user plane traffic and signaling separation is:

$$
\begin{gathered}
P_{b}=\frac{\frac{A^{C}}{C !}}{\sum_{i=0}^{C} \frac{A^{i}}{i !}}+\frac{\frac{A^{C-1}}{(C-1) !}}{\sum_{i=0}^{C} \frac{A^{i}}{i !}}+\cdots+ \\
+\frac{A^{C-S} /(C-S) !}{\sum_{i=0}^{C} A^{i} / i !}
\end{gathered}
$$

This formula describes a system, where the total number of servers is $C$, and we have blocking when at least $C-S$ resources are busy. Applying (9) on blocking probability on $\mathrm{S} 5$ interface (8):

$$
\begin{gathered}
P_{b, L Q S I G}=\frac{\frac{A_{L Q S I G}^{c_{3}}}{c_{3} !}}{\sum_{i=0}^{c_{3}} \frac{A_{L Q S I G}^{i}}{i !}}+\frac{A_{L Q S I G}^{c_{3}-1} /\left(c_{3}-1\right) !}{\sum_{i=0}^{c_{3}} \frac{A_{L Q S I G}^{i}}{i !}}+\cdots+ \\
+\frac{A_{L Q S I G}^{c_{3}-S} /\left(c_{3}-S\right) !}{\sum_{i=0}^{C_{3}} A_{L Q S I G}^{i} / i !}
\end{gathered}
$$




\section{B. Legacy Model}

In order to measure the performance of our newly introduced LQSIG resource reservation protocol, it is obvious to compare the calculated blocking probability to the standard LTE networks. Two different architectural points are important to be emphasized, where resource reservation makes some simplification. The real data path can be seen in Fig. 4, as a dotted line.

Connections of eNodeBs to the Cell Site Routers are not always direct; the base stations are usually chained after each other with microwave links typically. Such a chain can be either a ring or a tree-like structure, may be a hybrid topology. A ring can be used for backup or load balance purposes. It can be proved easily, that a ring has a worse blocking probability, in case eNodeBs are identical. In this analysis we do not assume a ring, but only tree structures. As an approximation, we use three tree levels; the farther-most eNodeB is connected with three links to the Cell Site Router via other two eNodeBs in the data path. [14]

The other important part of the architecture of the backhaul network, which is transparent when using LQSIG. In legacy LTE networks it has a hierarchical topology: eNodeBs are connected to the Cell Site Routers; their traffic is going to the Aggregation Site Routers. Core Site Routers are used as gateways towards the LTE core Networks. In this order they all have more interfaces and a higher throughput capacity [15].

In our analysis we assume:

- 3 cells are served by each eNodeB

- 300 eNodeBs, 100 on each tree level

- Links of eNodeB have capacity of $150 \mathrm{Mbps}, 300$ Mbps and 1 Gbps respectively (the last one is connected to the Cell Site)

- 50 Cell Sites, 5 connected to an Aggregation Site with 10

- 10 Aggregation Sites, 2 connected to a Core Site with 40 Gbps

- 5 Core Sites, 5 connected to a SGW with 80 Gbps

- $1 \mathrm{SGW}$, connected to 1 PDN-GW with $100 \mathrm{Gbps}$.

- One traffic class, typified with bandwidth requirement, 2 Mbps calls.

- The network can serve 50000 parallel calls, and further capacity of 500 calls are dedicated for signaling only.

A session is defined as a sequence of client/server interactions initiated by a single client for the duration of some work unit. The term session is protocol specific and has different meanings/demarcations for different levels in the protocol stack. From this point, the term call is defined as a session served by the network, having $2 \mathrm{Mbps}$ bandwidth requirement throughout the session time.

While calculating the blocking probability we are using the previously introduced hierarchical approach. Using formula (1) the offered traffic on level $i=0$ :

$$
A_{0}=\frac{\lambda_{0}+\lambda_{h, 0}}{\mu+n_{0}}
$$

where the offered traffic $A_{0}$ consists of the traffic due to new arrivals, the traffic due to handoffs.

Now we have nine levels in the tree structure, $i=0 \ldots 8$. After executing the iteration steps, we have blocking probability on S5 interface:

$P_{b, L T E}=\frac{\frac{A_{L T E}^{c_{8}}}{c_{8} !}}{\sum_{j=0}^{c_{8}} \frac{A_{L T E}^{j}}{j !}}+\frac{A_{L T E}^{c_{8}-1} /\left(c_{8}-1\right) !}{\sum_{j=0}^{c_{8}} \frac{A_{L T E}^{j}}{j !}}+\cdots+\frac{A_{L T E}^{c_{8}-S} /\left(c_{8}-S\right) !}{\sum_{j=0}^{c_{8}} A_{L T E}^{j} / j !}$

and the offered traffic

$$
A_{L T E}=\frac{N \lambda_{0}(1-P)}{\mu}
$$

where

$$
\begin{gathered}
N=N_{0} N_{1} N_{2} N_{3} N_{4} N_{5} N_{6} \\
1-P=\left(1-P_{b, 0}\right)\left(1-P_{b, 1}\right)\left(1-P_{b, 2}\right)\left(1-P_{b, 3}\right) * \\
*\left(1-P_{b, 4}\right)\left(1-P_{b, 5}\right)\left(1-P_{b, 6}\right)
\end{gathered}
$$

\section{Simulation Results}

Based on the above mentioned network parameters, using formula (7) and (10) we can calculate the blocking probability of S5 interface while using LQSIG resource reservation. We assume two kinds of typical calls, specifying two different call durations. The first is $10 \mathrm{~s}$, modelling the download time of a webpage, calculated with ETSI Kepler Reference page and typical downlink throughput values of an LTE network. The second is 5 minutes, modelling a video clip duration. It depends on optimizer platforms, whether 5 minutes is the total duration of the video and the mediation platform uses justin-time delivery, or video is longer, but a higher download speed enables to get the whole content at the playbacks beginning.

\begin{tabular}{c|c} 
TABLE 3: PARAMETERS OF LQSIG \\
\hline$N_{0}$ & 900 \\
$N_{1}$ & 300 \\
$C_{0}$ & 50 \\
$C_{1}$ & 150 \\
$C_{3}$ & 50000 \\
$S_{x}$ & $0,01 * C_{x}$ \\
$\mu_{1}$ & $1 / 10$ \\
$\mu_{2}$ & $1 / 600$ \\
$d$ & $45 \mathrm{~ms}$ \\
\hline
\end{tabular}

Now we need to define the parameters for calculating blocking probability in case of legacy LTE network, using formulas (12), (13), (14) and (15). Based on the assumed network topology:

TABLE 4: PARAMETERS OF LEGACY LTE

\begin{tabular}{cc|cc}
\hline$N_{0}$ & 900 & $C_{1}$ & 150 \\
$N_{1}$ & 100 & $C_{2}$ & 150 \\
$N_{2}$ & 100 & $C_{3}$ & 150 \\
$N_{3}$ & 100 & $C_{4}$ & 1000 \\
$N_{4}$ & 50 & $C_{5}$ & 5000 \\
$N_{5}$ & 10 & $C_{6}$ & 10000 \\
$N_{6}$ & 5 & $C_{8}$ & 50000 \\
$C_{0}$ & 50 & $\mu_{1}$ & $1 / 10$ \\
& & $\mu_{2}$ & $1 / 600$ \\
\hline
\end{tabular}

In the calculation we use the recursive version of Erlang-B formula, as the parameters are high and it is difficult to calculate the factorial of a high value.

$$
P_{x}=\frac{A * P_{x-1}(A)}{x+A * P_{x-1}(A)}
$$

where $A$ is offered traffic and $x$ is the number of parallel resources. 


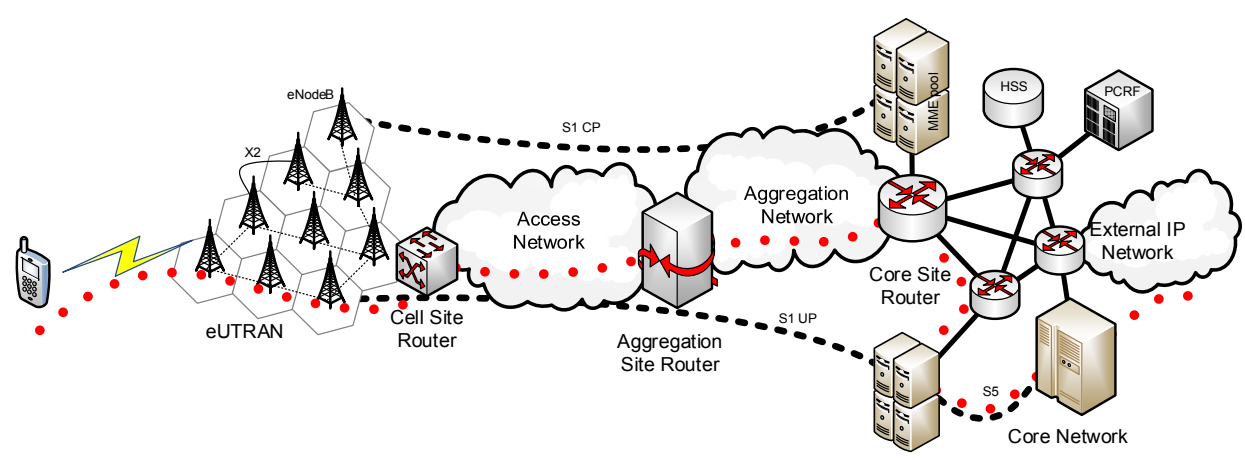

Fig. 4.Legacy LTE Architecture with data path.

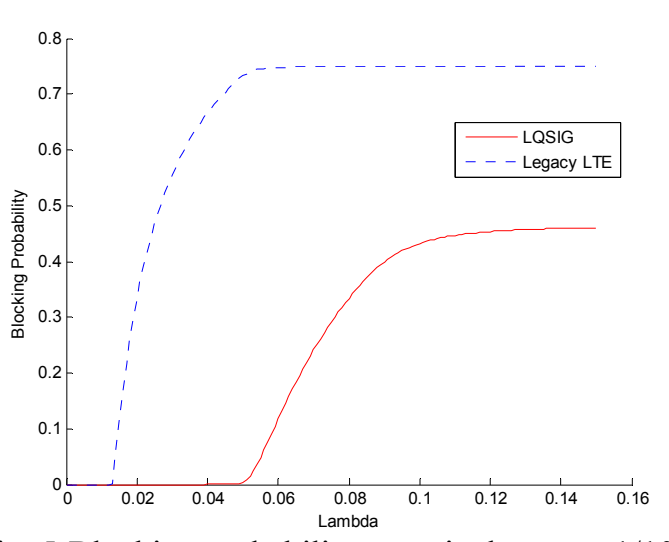

Fig. 5. Blocking probability vs arrival rate, $\mu=1 / 10$.

As shown in Fig. 5, LQSIG is able to present a better blocking probability of requested sessions compared to the case when resource reservation is not in use. This is so largely because resources of an old path are released immediately after the new EPS bearer is built up, optimal use of the network resources.

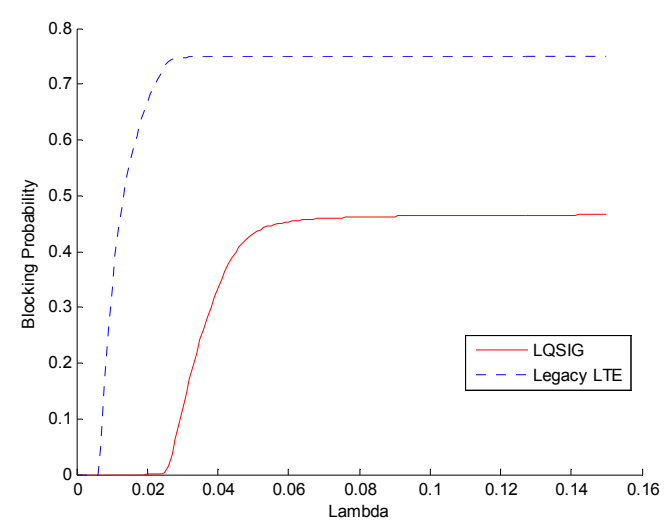

Fig. 6. Blocking probability vs arrival rate, $\mu=1 / 300$

The blocking probability according to the call arrival rate when a call duration is longer $(300 \mathrm{sec})$ is shown in Fig. 6. As the call arrival rate increases, legacy LTE blocking probability also dramatically increases. LQSIG tries to release the stale connections after handover, and thus the blocking probability of requested session in LQSIG scenarios is lower than that in legacy case.

\section{CONCLUSION}

QoS is a critical issue in the operation of today's telecom networks. In this paper we have identified the need for providing end-to-end QoS in LTE networks.
Based on the findings, we have proposed a LTE mobilityaware QoS signaling protocol (LQSIG) with key features such as QSPEC to LTE QoS class mapping, interworking with LTE CP protocol stack, emphasizing the hybrid solution with RRC extension. The proposed analytical model is used to determine the end-to-end blocking probability of LTE networks with LQSIG in use. The performance evaluation - presented in the last section has highlighted that the usage of resource reservation has an advantage compared to standard LTE in terms of blocking probability. Our research plans will include the introduction of more traffic classes into our analytical model.

\section{REFERENCES}

[1] H. Ekström, "QoS Control in the 3GPP Evolved Packet System", IEEE Communications Magazine, Vol. 47, Issue 2. pp. 76-83, February 2009.J. Clerk Maxwell, A Treatise on Electricity and Magnetism, 3rd ed., vol. 2. Oxford: Clarendon, 1892, pp.68-73.

[2] M. Marchese, "QoS over Hetergenous Networks", John Wiley \& Sons Ltd, 2007

[3] R. Nossenson, "Long Term Evolution Network Architecture", IEEE COMCAS November 2009.

[4] L. Li, S. Shen and C. Chen-Yui Yang, "LTE CoS/QoS Harmonization Emulator", IEEE CyberC, pages 154-161, October 2011.

[5] S. Sesia, I. Toufik and M. Baker, "LTE - The UMTS Long Term Evolution From Theory to Practice", John Wiley \& Sons Ltd, 2011

[6] H. Lee, S. Lee, B. Lee and J. Bang, "A Next Generation QoS Signaling Protocol for IP-based Wireless/Mobile Networks", Samsung Tech. Conference, 2005

[7] R. Bless and M. Röhricht, "QoS Support for Mobile Users using NSIS", Networking 2009, Lecture Notes in Computer Science, Vol. 5550, pp. 169-181, 2009

[8] J. Manner, G. Karagiannis and A. McDonald, "NSIS Signaling Layer Protocol (NSLP) for Quality-of-Service Signaling", RFC5974, IETF, October 2010.

[9] G. Ash, A. Bader, C. Kappler and D. Oran, "QSPEC Template for the Quality-of-Service NSIS Signaling Layer Protocol (NSLP)", RFC 5975, IETF, October 2010.

[10] 3GPP Tech. Spec. 25.331, "Radio Resource Control (RRC); Protocol Specification", v. 11.5.0

[11] S. Paskalis, A. Kaloxylos, E. Zervas and L. Merakos, "An Efficient RSVP - Mobile IP Interworking Scheme", Mobile Networks and Applications 8, pages 197-207, 2003

[12] G. Horváth, "End-to-end QoS Management Across LTE Networks", SoftCOM Conference, 2013

[13] G. Horváth, P. Fazekas, "End-to-end QoS Signaling for LTE", TELFOR Conference, 2013

[14] H. Lehpamer, "Microwave Tranmission Networks", McGraw-Hill, 2010

[15] Tellabs, "Unified Mobile Backhaul Architecture for LTE, 3G and 2G", $\quad$ http://www.tellabs.com/solutions/mobilebackhaul/tlab unified_backhaul_arch_sb.pdf 